\title{
Genome-wide identification and analysis of MAPK and MAPKK gene family in Chinese jujube (Ziziphus jujuba Mill.)
}

\author{
Zhiguo Liu ${ }^{1 \dagger}$, Liman Zhang ${ }^{2 \dagger}$, Chaoling Xue ${ }^{2}, \mathrm{Hu} \mathrm{Fang}^{3}$, Jin Zhao ${ }^{2^{*}}$ and Mengjun Liu ${ }^{1 *}$
}

\begin{abstract}
Background: Chinese jujube (Ziziphus jujuba Mill.) is one of the most important members in the Rhamnaceae family. The whole genome sequence and more than 30,000 proteins of Chinese jujube have been obtained in 2014. Mitogenactivated protein kinase cascades are universal signal transduction modules in plants, which is rapidly activated under various biotic and abiotic stresses. To date, there has been no comprehensive analysis of the MAPK and MAPKK gene family in Chinese jujube at the whole genome level.

Results: By performing a series of bioinformatics analysis, ten MAPK and five MAPKK genes were identified from the genome database of Chinese jujube, and then compared with the homologous genes from Arabidopsis. Phylogenetic analysis showed that ZjMAPKs was classified into four known groups, including A, B, C and D. ZjMAPKs contains five members of the TEY phosphorylation site and five members with the TDY motif. The ZjMAPKK family was subsequently divided into three groups, A, B and D. The gene structure, conserved motifs, functional annotation and chromosome distribution of ZjMAPKs and ZjMAPKKs were also predicted. ZjMAPKs and ZjMAPKKs were distributed on nine pseudochromosomes of Chinese jujube. Subsequently, expression analysis of ZjMAPK and ZjMAPKK genes using reverse transcription PCR and quantitative real-time PCR was carried out. The majority of ZjMAPK and ZjMAPKK genes were expressed in all tested organs/tissues with considerable differences in transcript levels indicating that they might be constitutively expressed. Moreover, ZjMKK5 was specific expressed in early development stage of jujube flower bud, indicating it plays some roles in reproductive organs development. The transcript expression of most ZjMAPK and ZjMAPKK genes was down-regulated in response to plant growth regulators, darkness treatment and phytoplasma infection.

Conclusions: We identified ten ZjMAPK and five ZjMAPKK genes from the genome database of Chinese jujube, the research results shown that ZjMPKs and ZjMKKs have the different expression patterns, indicating that they might play different roles in response to various treatments. The results provide valuable information for the further elucidation of physiological functions and biological roles of jujube MAPKs and MAPKKs.
\end{abstract}

Keywords: Chinese jujube, MAPKs, MAPKKs, Bioinformatics analysis, Gene expression

\footnotetext{
*Correspondence: zhaojinbd@126.com; Imj1234567@aliyun.com

†Equal contributors

${ }^{2}$ College of Life Science, Hebei Agricultural University, Baoding, China

${ }^{1}$ Research Center of Chinese Jujube, Hebei Agricultural University, Baoding,

China

Full list of author information is available at the end of the article
} 


\section{Background}

Mitogen-activated protein kinases (MAPK) and mitogenactivated protein kinases kinases (MAPKK) are significant families of serine/threonine kinases, which have been found to involve in wide variety of metabolism pathway include cell division, developmental processes and defense response [1-5]. MAPK cascades comprise three kinds of kinases: MAPK kinase kinase (MAPKKK/MEKK), MAPK kinase (MAPKK/MEK) and MAPK (MPK) [6], and they are gradually phosphorylated by upstream signal stimuli. MAPK substrates play important roles in regulating various intracellular reactions and responding to extra stimulation.

To date, the most extensively studied plant MAPK cascades genes are in Arabidopsis. After the completion of the Arabidopsis whole genome sequencing, 80 MAPKKKs, 10 MAPKKs and 20 MAPKs were screened firstly at genome level [7, 8]. Then, the members of MAPK cascades from rice, maize, Brachypodium distachyon, tobacco, Brassica rapa, Gossypium raimondii, poplar, apple, grape and mulberry at whole genome level were successively identified as well [9-18]. More bioinformatics and function studies of MAPK cascades genes were further carried out in these plants. Plant MAPKs (MPKs) can be divided into four groups (Group A, B, C and D) based on sequence alignment and the phylogenetic analysis, which have two phosphorylation motifs of TEY and TDY. Groups A, B and C of MAPKs share the TEY motif, while group D have the TDY motif and a long $C$ terminal sequence [7]. MAPKKs (MKKs) were also composed of four groups (Group A, B, C and D) in Arabidopsis thaliana and rice [19]. Although the structure of plant MAPK cascades genes is highly conserved, they have been proved to involve in various biotic and abiotic stresses, hormones, cell division, plant growth and developmental processes. For example, in Arabidopsis, AtMPK3 and AtMPK6 have been demonstrated to be the significant regulators in response to pathogen infections and abiotic stresses [20], while AtMPK9 and AtMPK12 were highly expressed in guard cells and regulated ROS-mediated ABA signaling positively [21]. In addition, overexpressing GhMPK16 in Arabidopsis has been indicated that it have significant resistance to bacterial pathogens, fungi, drought and $\mathrm{H}_{2} \mathrm{O}_{2}$ [22].

Chinese jujube (Ziziphus jujuba Mill.) has experienced more than 7000 years in artificial cultivation, which is one of the most important member in the Rhamnaceae family [23]. Its fruit is rich in vitamin C and sugar [24]. Moreover, the jujube trees have stronger adaption to various biotic and abiotic stresses, especially drought and salinity. The whole genome sequence and more than 32,000 proteins of Chinese jujube have been obtained recently [25], which provided data resource for genomewide analyses of specific gene families. To date, there have been no studies into the jujube MAPK and
MAPKK gene family at the whole genome level. The information of MAPKs and MAPKKs of Chinese jujube is also very limited in the public databases.

Taking advantage of the available jujube genome database, a genome-wide search for the homologues of the MAPK and MAPKK families in jujube was performed in this study. Five MAPKK and ten MAPK genes were identified from jujube genome. The phylogenetic analysis, gene structure, conserved motifs, functional annotation and chromosome distribution of ZjMAPKs and ZjMAPKKs were also predicted. In addition, expression profile analyses of above genes using the reverse transcription PCR (RT-PCR) and quantitative real-time PCR (qRT-PCR) were performed in different materials.

\section{Methods}

Plant materials, growth conditions and treatments

Seven different tissue samples (root, bearing shoot, secondary shoot, leaf, flower bud, flower, and fruit) were collected separately from three trees and used for organ-specific expression analysis. The floral organs were collected at 7 different development stages for qRT-PCR expression analysis, as it shown in Additional file 1: Figure S1.

The cultivar of plantlet which used for plant hormone and darkness experiment was Ziziphus jujuba Mill. 'Dongzao'. All plantlets were collected from Research Centre of Chinese Jujube, Hebei Agricultural University and cultured in a growth cabinet with a photoperiod of $16 \mathrm{~h}$ artificial light and $8 \mathrm{~h}$ darkness at $26 \pm 2{ }^{\circ} \mathrm{C}$.

For $\alpha$-Naphthalene acetic acid (NAA), indole-3-butyric acid (IBA) and 6-benzyladenine (6-BA) treatments, the plantlets were subjected to $0.5 \mathrm{mg} / \mathrm{L}$ IBA, $1 \mathrm{mg} / \mathrm{L} 6-\mathrm{BA}$ and $1 \mathrm{mg} / \mathrm{L} \mathrm{NAA}$, respectively. Plantlets incubated on Murashige and Skoog (MS) medium without plant growth regulators were used as control. All the samples for RNA extractions were collected at 20 days after treatments. For darkness treatment, the plantlets were cultured in a dark chamber and collected at $0 \mathrm{~d}, 4 \mathrm{~d}, 8 \mathrm{~d}, 12 \mathrm{~d}$ and $16 \mathrm{~d}$ after darkness treatment for RNA isolation. The samples with 0 d darkness treatment were used as control.

For the biotic stress treatment, 'Dongzao' plantlets infected with jujube witches' broom (JWB) were used as test group. JWB, caused by phytoplasma, is the most serious and destructive disease for jujube tree cultivation. At the same time, healthy plantlets of 'Dongzao' were used as control group. All plantlets were cultured in MS medium without plant growth regulators.

Three biological replicates were collected in each treatment. The samples were stored at $-80{ }^{\circ} \mathrm{C}$ for RNA extraction and expression analysis.

\section{Cloning of the ZjMPK4 gene}

ZjMPK4 partial gene sequence was identified from Chinese jujube genome data. So, homology-based cloning method 
was used to isolate ZjMPK4 coding sequence (CDS) from Chinese jujube. According to the gene sequences of Prunus persica and Prunus mume, Primer Premier 5.0 software was used to design specific primers. The ZjMPK4 genespecific primers as follow: ZjMPK4-S: 5' - ATGGCTG CCAAGGAGTCAAG-3', ZjMPK4-A: 5' - CTAATGGATT GGGGGTCTGG-3'.

Identification of ZjMAPKs and ZjMAPKKs in Chinese jujube To identify potential members of the MAPK and MAPKK gene family in Chinese jujube, the completed jujube genome sequence from the DDBJ/EMBL/GenBank (accession JREP00000000) [25] was used. MAPKs and MAPKKs genes of Arabidopsis (All sequences were shown in Additional file 2) were used as queries to search in the whole jujube genome database firstly. After searching for MAPK and MAPKK gene family, the Pfam (http://xfam.org/) and SMART (http://smart.embl-heidelberg.de/) [26] databases were used to confirm that the predicted jujube MAPK and MAPKK proteins. Finally, those genes containing full open reading frame (ORF) were manually analyzed using InterProScan and ClustalX program to confirm conserved domains or motifs of MAPK and MAPKK.

\section{Gene structure and protein structure analysis of ZjMAPKs} and ZjMAPKKs

National Center for Biotechnology Information (NCBI, http://www.ncbi.nlm.nih.gov) ORF finder was used to find putative open reading frames and functional domains were determined by blastp of NCBI as well. The gene structures of the ZjMAPKs and ZjMAPKKs were generated with the GSDS (Gene Structure Display
Server: http://gsds.cbi.pku.edu.cn/). Then, the exonintron structures for individual jujube ZjMAPK and ZjMAPKK genes were checked and measured by aligning the cDNA sequences to their corresponding genomic DNA sequences [27]. The isoelectric points and molecular weights of ZjMAPKs and ZjMAPKKs were obtained with the help of proteomics and sequence analysis tools on the ExPASy Proteomics Server (http://expasy.org/). All of the relevant genes identified in the jujube genomes were aligned using ClustalX program (version 2.0).

\section{The chromosomal location of ZjMAPKs and ZjMAPKKs}

To determine the locations of the two gene families on pseudo-chromosomes, the ZjMAPKs and ZjMAPKKs sequences were further used as query sequences for the BLASTN search against the jujube genome sequence. Each ZjMAPKs and ZjMAPKKs gene was mapped to the jujube genome according to our previously study [28].

\section{Multiple sequence alignment and phylogenetic tree construction}

The full-length amino acid sequences of the MAPKs (20 AtMAPKs, 10 ZjMAPKs) and MAPKKs (10 AtMAPKKs, 5 ZjMAPKKs) were used for multiple sequence alignment and phylogenetic analyses, respectively. Multiple sequence alignments of the amino acid sequences were conducted with default parameters by using ClustalX program (version 2.0). Phylogenetic analyses based on the protein sequences were performed by the software MEGA5.0 [29]. The unrooted phylogenetic trees were constructed using the neighbor-joining (NJ) method, and the reliability of the trees obtained was tested using

Table 1 The information of MAPK and MAPKK gene family in Chinese jujube

\begin{tabular}{|c|c|c|c|c|c|c|c|c|c|c|}
\hline Gene family & Gene name & Gene model & Chromosomes & Position & ORF (bp) & Size (aa) & $\mathrm{MW}(\mathrm{KD})$ & $\mathrm{Pl}$ & Types & Group \\
\hline \multirow[t]{10}{*}{ MAPK } & ZjMPK1 & CCG019246.1 & Chr1 & scaffold501:141,704:145,538:+ & 1197 & 398 & 45.45 & 5.57 & TEY & A \\
\hline & ZjMPK2 & CCG014282.1 & Chr2 & scaffold312:291,457:294,343:- & 1128 & 375 & 42.96 & 5.61 & TEY & A \\
\hline & ZjMPK3 & CCG004076.1 & Chr12 & scaffold130:145,282:154,186:+ & 1497 & 498 & 57.29 & 6.13 & TEY & B \\
\hline & ZjMPK4 & & & & 1140 & 379 & 43.36 & 6.36 & TEY & B \\
\hline & ZjMPK5 & CCG015764.1 & Chr8 & scaffold364:308,649:310,334:+ & 1125 & 374 & 43.36 & 7.60 & TEY & C \\
\hline & ZjMPK6 & CCG007165.2 & Chr3 & scaffold164:60,108:64,064:- & 1728 & 575 & 64.79 & 9.06 & TDY & $\mathrm{D}$ \\
\hline & ZjMPK7 & CCG004734.3 & Chr4 & scaffold1368:71,249:76,280:- & 1851 & 616 & 70.24 & 9.10 & TDY & $\mathrm{D}$ \\
\hline & ZjMPK8 & CCG026115.1 & Chr7 & scaffold828:71,756:83,030:+ & 2166 & 721 & 81.15 & 7.82 & TDY & $\mathrm{D}$ \\
\hline & ZjMPK9 & CCG022866.1 & Chr1 & scaffold663:208,540:214,001:+ & 1746 & 581 & 66.68 & 7.28 & TDY & D \\
\hline & ZjMPK10 & CCG016360.1 & & scaffold389:214,895:246,410:+ & 1896 & 631 & 71.83 & 6.66 & TDY & $\mathrm{D}$ \\
\hline \multirow[t]{5}{*}{ MAPKK } & ZjMKK1 & CCG011783.1 & Chr8 & scaffold25:290,193:292,650:+ & 1062 & 353 & 39.19 & 5.53 & & A \\
\hline & ZjMKK2 & CCG011406.1 & Chr12 & scaffold24:794,781:798,772:+ & 1065 & 354 & 39.50 & 6.02 & & A \\
\hline & ZjMKK3 & CCG021919.1 & Chr4 & scaffold612:169,916:171,832:+ & 1008 & 335 & 36.96 & 5.76 & & B \\
\hline & ZjMKK4 & CCG028907.1 & Chr11 & scaffold99:188,221:189,240:+ & 1020 & 339 & 37.78 & 5.96 & & $\mathrm{D}$ \\
\hline & ZjMKK5 & CCG005893.1 & Chr5 & scaffold1471:17,168:18,166:- & 999 & 332 & 36.70 & 6.88 & & $\mathrm{D}$ \\
\hline
\end{tabular}


bootstrapping with 1000 replicates. Scale bar represents 0.05 amino acid substitution per site.

\section{Reverse transcription PCR and quantitative real-time PCR analysis}

Total RNA was extracted using the TIANGEN RNA Extraction Kit. First-strand cDNA was synthesized using the PrimeScript 1st Strand cDNA Synthesis Kit (Takara, Dalian, China) according to manufacturer's instructions. The resulting cDNA was then diluted nine-fold and stored at $-20^{\circ} \mathrm{C}$ for the subsequent RT-PCR and qRT-PCR assays.

For gene expression quantification, specific primers were designed for each ZjMAPKs and ZjMAPKKs gene using the Primer Premier 5.0 software. The information of primers used in this study is listed in the Additional file 3: Table S1. The organ-specific expression analysis was assayed by RT-PCR. The conditions for RT-PCR were as following: $4 \mathrm{~min}$ at $94{ }^{\circ} \mathrm{C}$, followed by 30 cycles of $30 \mathrm{~s}$ at $94{ }^{\circ} \mathrm{C}, 30 \mathrm{~s}$ at $55-60{ }^{\circ} \mathrm{C}$ (varying with specific primers),

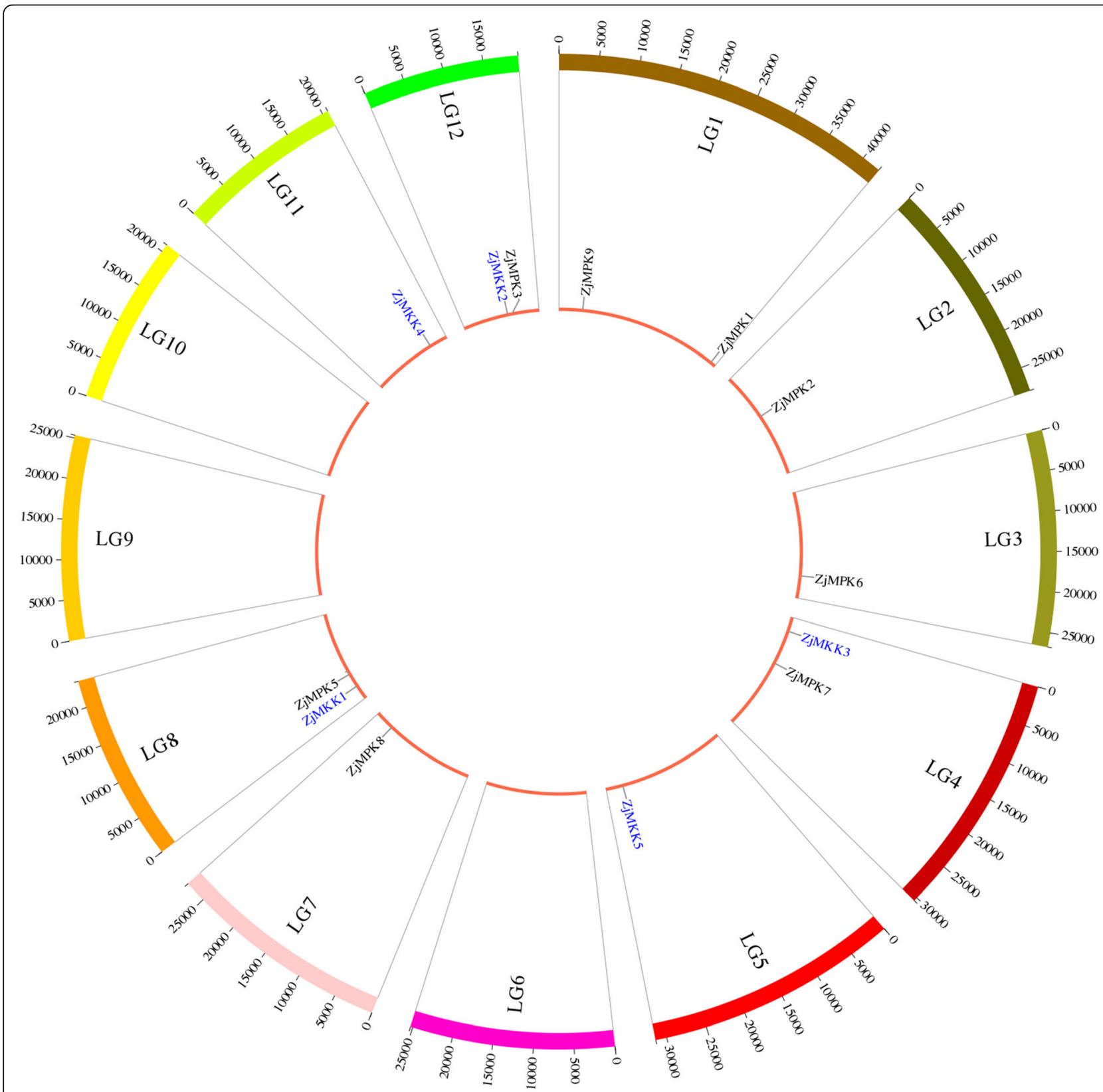

Fig. 1 Positions of ZjMPK and ZjMKK genes on the jujube chromosomes. Genes were mapped to the jujube chromosomes via the Circos tool. The jujube chromosomes were arranged in a circle 
and $45 \mathrm{~s}$ at $72{ }^{\circ} \mathrm{C}$, and a final extension at $72{ }^{\circ} \mathrm{C}$ for $7 \mathrm{~min}$ to complete the reaction.

The expression of ZjMAPKs and ZjMAPKKs genes in different treatments was examined using qRT-PCR. The qRT-PCR was performed on the Bio-Rad iQ $^{\text {tw }} 5$ using TransStart Top Green qPCR SuperMix AQ131 (TransGen Biotech, China). The $25 \mu \mathrm{L}$ reaction system contains $12.5 \mu \mathrm{L}$ of $2 \times$ TransStart Top Green qPCR SuperMix, $0.5 \mu \mathrm{L}$ each of $10 \mu \mathrm{M}$ primers, $1 \mu \mathrm{L}$ diluted cDNA and

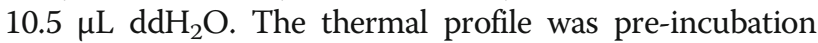
for $30 \mathrm{~s}$ at $95{ }^{\circ} \mathrm{C}$, followed by 40 cycles of $5 \mathrm{~s}$ at $95^{\circ} \mathrm{C}, 10 \mathrm{~s}$ at $55-60{ }^{\circ} \mathrm{C}$ (varying with specific primers), and $10 \mathrm{~s}$ at $72{ }^{\circ} \mathrm{C}$. Subsequently, a melting curve analysis was run for one cycle from $55{ }^{\circ} \mathrm{C}$ to $90{ }^{\circ} \mathrm{C}$. Three biological replicates were performed for each sample. To normalize the total amount of cDNA present in each reaction, the Chinese jujube $Z j A C T$ gene was co-amplified as an endogenous control for calibration of relative expression. The relative expression level was calculated by the $2^{-\triangle \triangle \mathrm{CT}}$ method [30].

\section{Statistical analysis}

Data are shown as the means \pm SD of independent experiments. Statistical analysis was performed by SPSS 16.0 software using student's t-test or ANOVA test method. $P<0.05$ and $P<0.01$ were considered as significant and highly significant difference, respectively.

\section{Results}

Identification of ZjMAPKs and ZjMAPKKs in Chinese jujube From jujube genome, nine typical MAPK genes and five typical MAPKK ones (Table 1) were identified and used for further analysis (All sequences were shown in Additional file 2). One MAPK gene (ZjMPK4) was isolated by homology cloning (The sequence was shown in Additional file 2). Sequence alignment of amino acid found that the sequence identity of Chinese jujube ZjMPK4 and Arabidopsis thaliana AtMPK4 was $82.85 \%$. The CDS length for ZjMAPK genes ranged from $1125 \mathrm{bp}$ (ZjMPK5) to $2166 \mathrm{bp}$ (ZjMPK8), and they encoded proteins ranging from 374 to 721 amino acids (aa) in length with an average of 515 aa, with a predicted molecular mass range of $42.63-81.15 \mathrm{KDa}$, and protein pIs ranging from 5.57 (ZjMPK1) to 9.10 (ZjMPK7) (Table 1). The CDS length for MAPKK genes ranged from 999 bp (ZjMKK5) to $1065 \mathrm{bp}(Z j M K K 2)$, and they encoded proteins ranging from 332 to 354 amino acids (aa) in length with an average of 342 aa, with a predicted molecular mass range of $36.70-39.50 \mathrm{KDa}$, and protein pIs ranging from 5.53 (ZjMKK1) to 6.88 (ZjMKK5) (Table 1). ZjMAPKs and ZjMAPKKs were randomly distributed on 9 chromosomes of Chinese jujube (Table 1 and Fig. 1).

\section{Conserved domains and motifs in ZjMAPKs and ZjMAPKKs}

Ten ZjMAPK and five ZjMAPKK underwent multiple sequence alignment by ClustalX program (Fig. 2), respectively. The result showed that all the ZjMAPKs and ZjMAPKKs that shared the family joint structure contained conserved protein kinase domains. A TXY motif phosphorylation site existed in ZjMAPKs, among them the TEY type existed in groups A, B and C, whereas group D contained the TDY motif (Fig. 2 and Table 1).

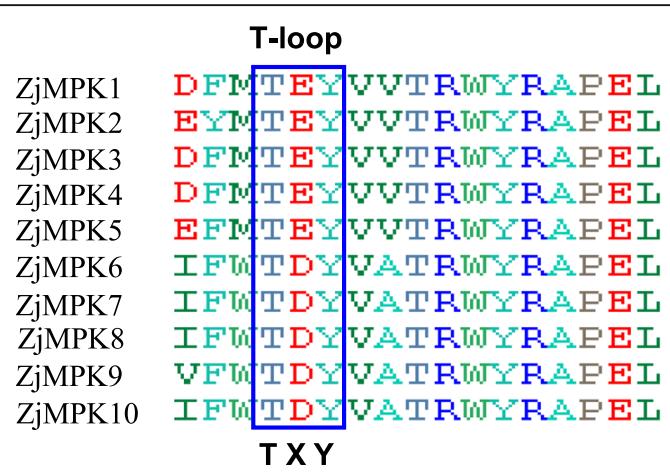

Conserved S/T-X5-S/T motif

ZjMKK1 GALERTGDTTLT

ZjMKK2 GASLA

ZjMKK3 GRGLEMVMMCATVUT

ZjMKK4 GDVUA TCETCD SMGT

ZjMKK5 GKIIETLDACNG VUT

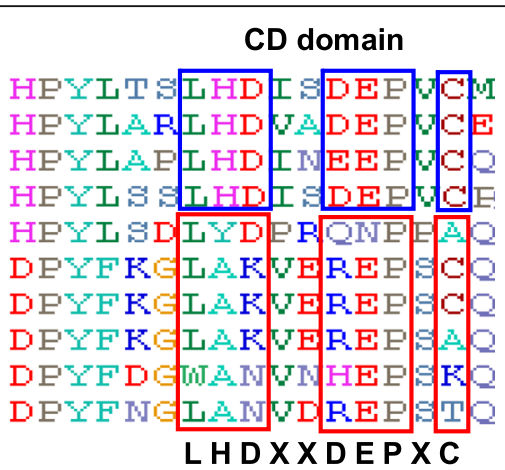

Active site $\mathrm{D}(\mathrm{I} / \mathrm{L} / \mathrm{V}) \mathrm{K}$ motif

KHIIHF DLAESHLIN

RHVIHADIHE GNLIVW

RHLVHPDIEEANLLVW

MOIVHP DIAE

KKIVHFDIEE BNLLUT

Fig. 2 Multiple sequence alignment of the kinase domains of ZjMPK and ZjMKK proteins 


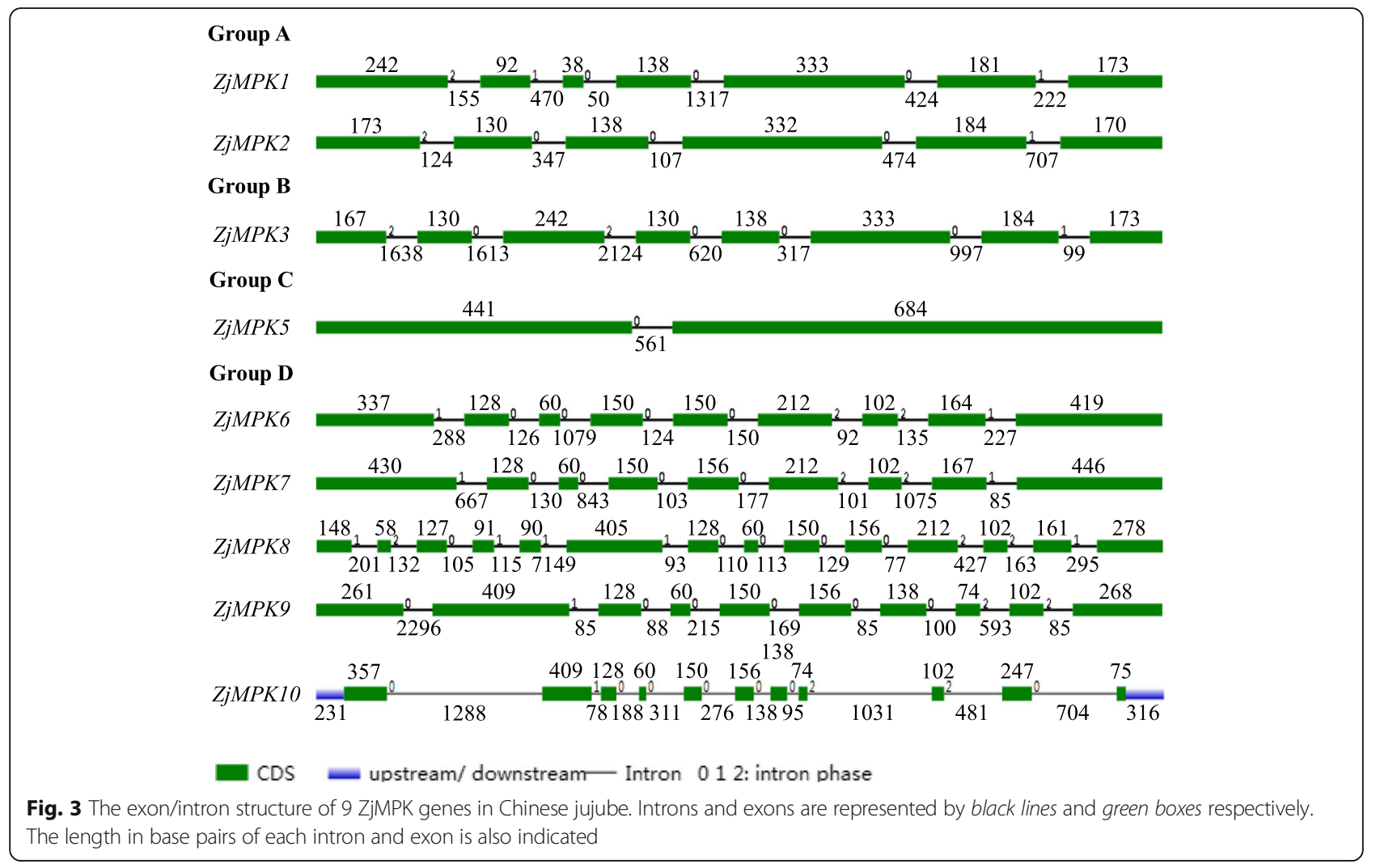

Two conserved motifs, S/T-X5-S/T and active site D (I/ $\mathrm{L} / \mathrm{V}) \mathrm{K}$, were found in all ZjMAPKKs (Fig. 2).

The gene structure of ZjMAPKs and ZjMAPKKs

The exon-intron structure diagram for the ZjMAPKs and ZjMAPKKs was also aligned and compared (Figs. 3 and 4). The different groups of ZjMAPKs and
ZjMAPKKs have different exon-intron structures, but most members within the same group shared a similar structure and gene length (Figs. 3 and 4). The number introns of ZjMAPKs were from 1 (ZjMPK5, one group $\mathrm{C}$ gene) to 13 (ZjMPK8 and ZjMPK10, two group D genes) (Fig. 3). In group A, ZjMPK1 and ZjMPK2 have seven and six exons, respectively, while all group A MAPKs in

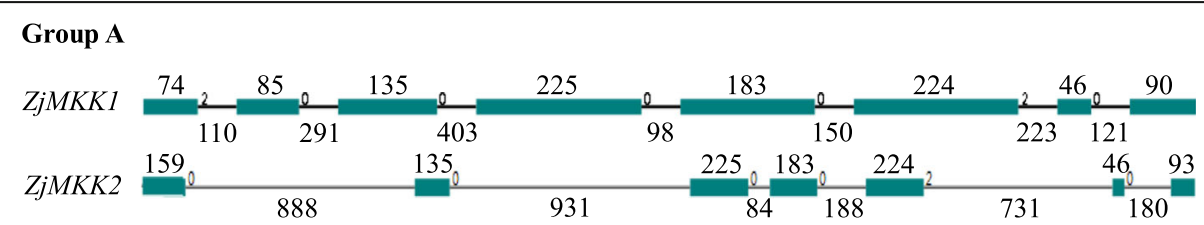

Group B

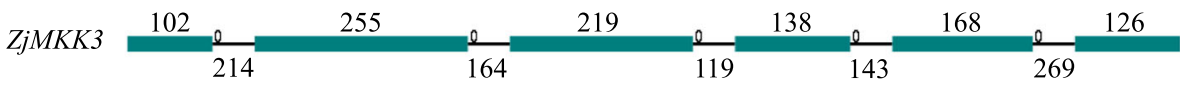

Group D

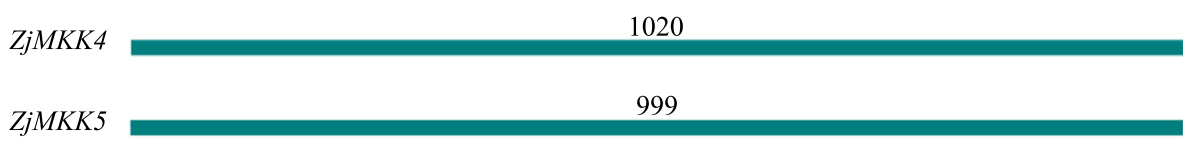

Exon - Intron 012 : intron phase

Fig. 4 The exon/intron structure of 5 ZjMKK genes in Chinese jujube. Introns and exons are represented by black lines and blue boxes respectively. Introns and exons are represented by black lines and green boxes respectively. The length in base pairs of each intron and exon is also indicated. Numbers correspond to the length of the intron and exon 
Arabidopsis, mulberry, poplar and tomato consisted six exons. Group B ZjMPK3 includes eight exons, which number is more than that of Arabidopsis, mulberry, poplar and tomato. Two group C ZjMAPK genes have two exons with strictly conserved sizes, which number are same to the other plant, including Arabidopsis, poplar, apple, mulberry and tomato. Moreover, Group C MAPKs of different plants also have similar gene length. The gene structure can provide some useful information of the genomic evolution. The conserved structural patterns of Group C MAPKs in different plants suggested that they may be conserved in evolution. Like other plants, Group D ZjMAPKs possessed a complex distribution of exons and introns, such as both ZjMPK8 and ZjMPK10 contained 14 exons.

The number introns of $Z j M A P K K s$ were from five to eight (Fig. 4), except the group D ZjMAPKKs. Members of group D ZjMAPKKs have a completely intron less structure, which is same with that in apple, poplar and Arabidopsis. In addition, the number of MAPKK members in all reported plants is always less than that of MAPKs and MAPKKKs, indicating that plant MAPKK genes may be more conserved in evolution than MAPKs and MAPKKKs.

\section{Multiple sequence alignment and phylogenetic tree construction}

In order to evaluate the evolutionary relationship among the MAPK and MAPKK proteins from different species, MAPKs (20 AtMAPKs, 10 ZjMAPKs) and MAPKKs (10 AtMAPKKs, 5 ZjMAPKKs) from Chinese jujube and Arabidopsis were subjected to a multiple sequence alignment using the MEGA 5.0 program based on their predicted amino acid sequences. The multiple sequence

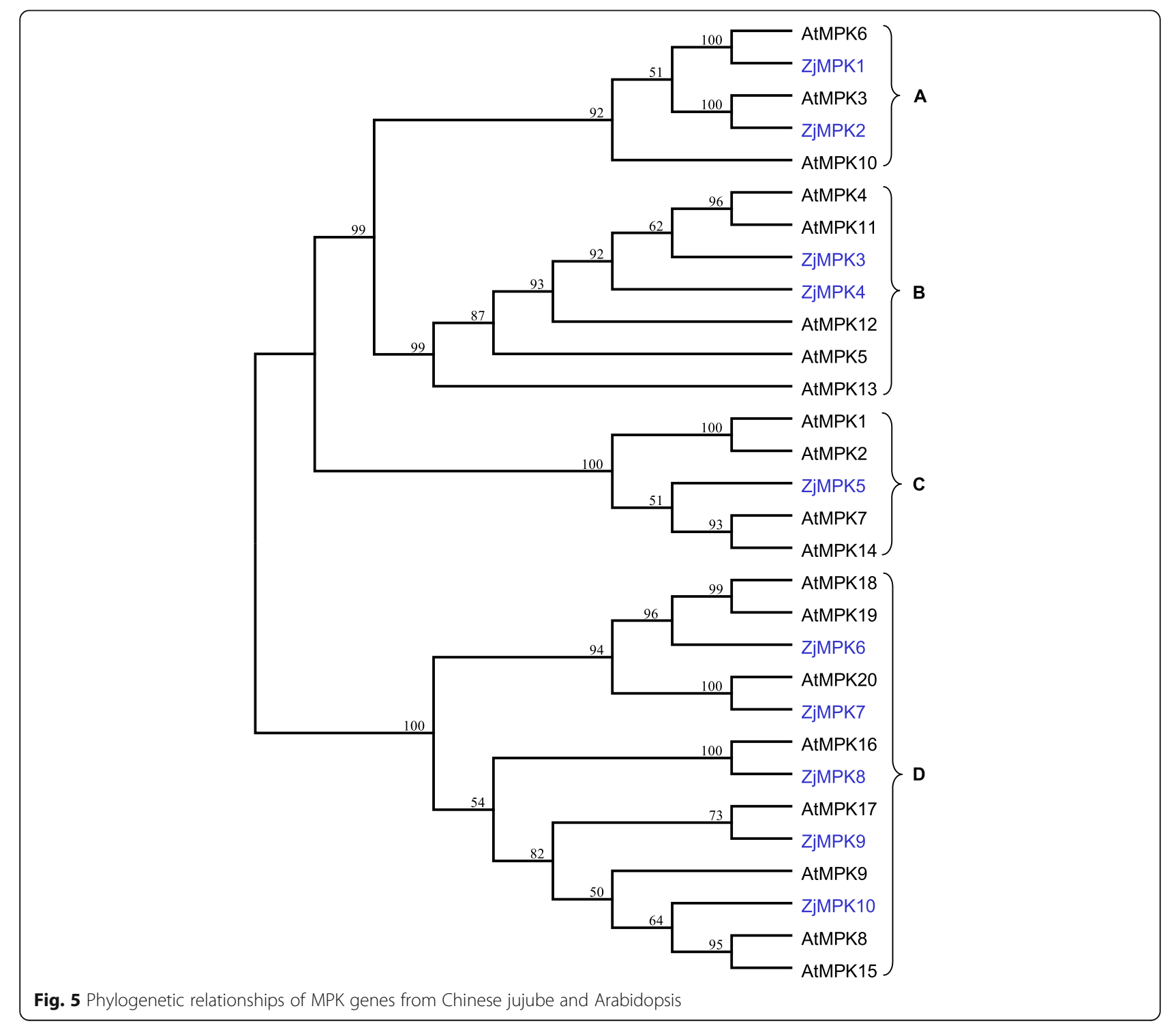


Table 2 The number of the MAPK and MAPKK gene family in Chinese jujube, Arabidopsis, Rice, Poplar, Tomato, Brachypodium distachyon, Apple, Mulberry and Maize

\begin{tabular}{|c|c|c|c|c|c|c|}
\hline Gene family & Species & Group A & Group B & Group C & Group D & Total \\
\hline \multirow[t]{9}{*}{ MAPK } & Arabidopsis & 3 & 5 & 4 & 8 & 20 \\
\hline & Rice & 2 & 1 & 2 & 10 & 15 \\
\hline & Poplar & 4 & 4 & 4 & 9 & 21 \\
\hline & Tomato & 3 & 4 & 2 & 7 & 16 \\
\hline & B. distachyon & 2 & 2 & 3 & 9 & 16 \\
\hline & Apple & 5 & 6 & 5 & 10 & 26 \\
\hline & Mulberry & 2 & 3 & 2 & 3 & 10 \\
\hline & Maize & 4 & 2 & 2 & 11 & 11 \\
\hline & Chinese jujube & 2 & 1 & 2 & 5 & 10 \\
\hline \multirow[t]{6}{*}{ MAPKK } & Arabidopsis & 3 & 1 & 2 & 4 & 10 \\
\hline & Rice & 2 & 1 & 2 & 3 & 8 \\
\hline & Poplar & 3 & 1 & 2 & 5 & 11 \\
\hline & B. distachyon & 2 & 3 & 2 & 5 & 12 \\
\hline & Apple & 3 & 1 & 2 & 3 & 9 \\
\hline & Chinese jujube & 2 & 1 & 0 & 2 & 5 \\
\hline
\end{tabular}

alignment file was then used for the construction of an unrooted phylogenetic tree. The phylogenetic analysis of the two species indicated that genes that were clustered together might have similar functions.

Through phylogenetic analyses of the two species, we found that the ZjMAPKs could also be classified into four groups corresponding to the groups A, B, C and D in Arabidopsis (Fig. 5). As shown in Fig. 5, the group D constitutes the largest clade containing 5 ZjMAPKs, followed by groups A ( 2 genes) and $C$ ( 2 genes), and the group B include one gene. As shown in Table 2, the group D gene family is the largest subfamily in Arabidopsis, maize, rice, Brachypodium distachyon, tomato, poplar and apple, too. The size of groups A, B and C is similar in rice, Brachypodium distachyon, maize and mulberry, including less genes than in Arabidopsis, tomato, poplar and apple, which shows that the amplification of groups A, B and C gene numbers is particularly impressive in the four species.

Only 5 MAPKK genes were identified from the Chinese jujube genome, and divided into three subfamilies (groups A, B and D) by the phylogenetic analysis (Fig. 6). ZjMKK1-2, ZjMKK3, and ZjPKK4-5 belong to groups $\mathrm{A}, \mathrm{B}$, and $\mathrm{D}$, respectively. In comparison with other plants (Arabidopsis, rice, Brachypodium distachyon, poplar and apple), it is found that the number of MAPKK gene (from 8 to12) is very conserved and MAPKKs can be divided into four groups $(\mathrm{A}, \mathrm{B}, \mathrm{C}$, and $\mathrm{D})$ in the other

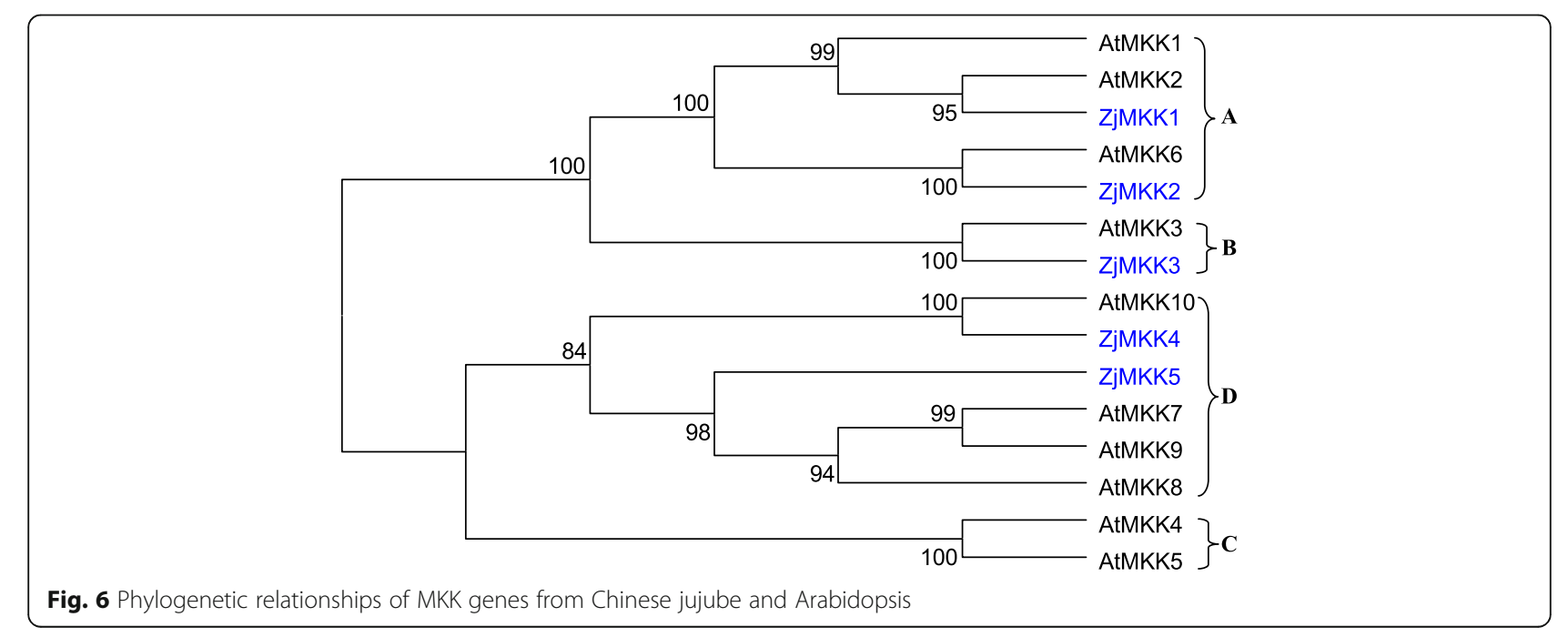


plants (Table 2), but ZjMAPKK genes in group C were not founded.

\section{Expression patterns of ZjMAPK and ZjMAPKK family genes}

Specific primer sets were designed for each ZjMAPKs and ZjMAPKKs (Additional file 3: Table S1) and their expression patterns in different materials were detected by semi-quantitative RT-PCR (Figs. 7, 8) and qRT-PCR (Figs. 9, 10 and 11). Most of the genes were expressed in all tested organs/tissues with considerable differences in transcript levels representing their distinct roles in jujube growth and development. ZjMKK2 was highly expressed in fruits and ZjMKK5 was specifically expressed in flower bud (Fig. 8), indicating their functions were involved in reproductive organs development. Moreover, ZjMKK5 was highly expressed in early stage of floral organ development which was further confirmed by qRT-PCR (Additional file 4: Figure S2). In addition, some genes of the same clade showed similar expression patterns, suggesting possible similar function or functional redundancy.

To study the function of the ZjMPKs and ZjMKKs in responding to abiotic stresses, the expression of these genes in response to different plant growth regulators and darkness treatment was examined by qRT-PCR. We found that the expression patterns of the two families were different when exposure to various plant growth regulators (Fig. 9). Moreover most members of the two family genes showed similar expressions in response to NAA and IBA treatments except for ZjMKK4, this may be due to both NAA and IBA are auxin analogues.

During the darkness treatment, the ZjMPK genes exhibited differential expression patterns (Fig. 10). The expression of ZjMPK7, ZjMPK8, ZjMPK9 and ZjMPK10

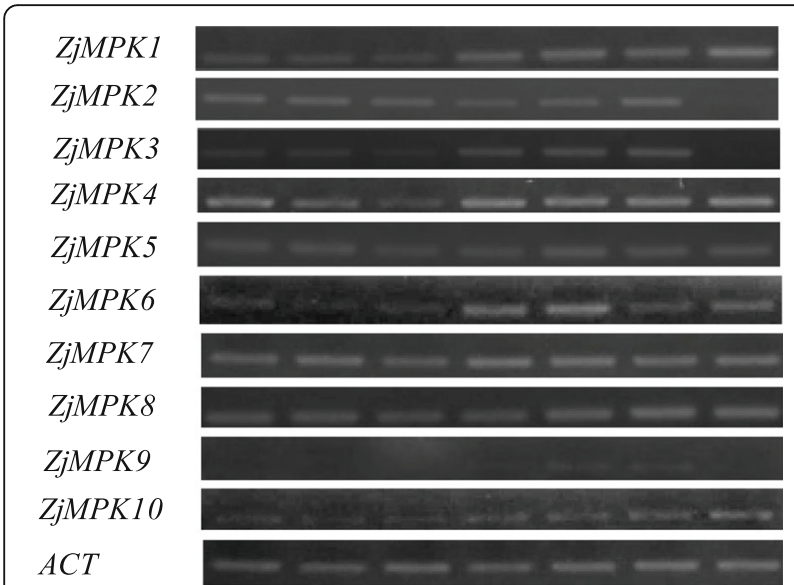

Fig. 7 Tissue-specific expression patterns for ZjMPKs. ZjACT was used as an internal control. From left to right: root, bearing shoot, secondary shoot, leaf, flower bud, flower, and fruit

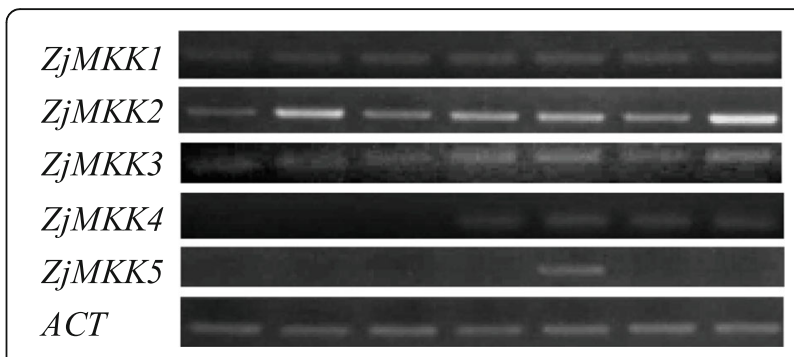

Fig. 8 Tissue-specific expression patterns for ZjMKKs. ZjACT was used as an internal control. From left to right: root, bearing shoot, secondary shoot, leaf, flower bud, flower, and fruit

was decreased significantly at the early stage, and then remained at the low level at the following stage in response to darkness treatment. However, ZjMPK1, $Z j M P K 2$, and ZjMPK4 expression were significant decrease from $4 \mathrm{~d}$ to $12 \mathrm{~d}$ during the darkness treatment, while increased at last stage. ZjMPK5 expression decreased sharply at first two stages, but there have no obvious changes in followed stage. The expression of $Z j M P K 3$ was decreased gradually in response to darkness treatment, while the transcript level of ZjMPK6 was significantly increased at 4 days after darkness treatment, and then markedly decreased, but later increased again. The ZjMKK genes also exhibited distinct expression profiles in response to darkness treatment (Fig. 10). Three $Z j M K K$ genes were down-regulated and only one gene was up-regulated as compared to the control in the darkness treatment. ZjMKK2 and ZjMKK3 displayed the similar expression patterns with ZjMPK5 and ZjMPK10, respectively. Additionally, ZjMKK4 expression profile was the same as ZjMPK3. Moreover, ZjMKK1 and ZjMPK6 exhibited the same expression patterns.

Evidence is accumulating that MAPK and MAPKK proteins are involved in responding to various biotic stresses. Thus, the expressions of these genes were examined in diseased plantlets infected by phytoplasma. As shown in Fig. 11, most of the ZjMPK genes were down-regulated under phytoplasma infection. Specially, the expression of ZjMPK1, ZjMPK3, ZjMPK4 and $Z j M P K 9$ was decreased dramatically. The expression level of $Z j M K K 1$ and $Z j M K K 3$ in diseased plantlets was decreased significantly, but the expression of ZjMKK2 was increased.

Overall, ZjMPKs and ZjMKKs display the different expression patterns, indicating that they may play different roles in response to various treatments.

\section{Discussion}

So far, MAPK and MAPKK gene families have been identified and functionally characterized in many plants, such as 20 MAPKs and 10 MAPKKs in Arabidopsis, 15 MAPKs and 8 MAPKKs in rice, 26 MAPKs and 9 
NAA

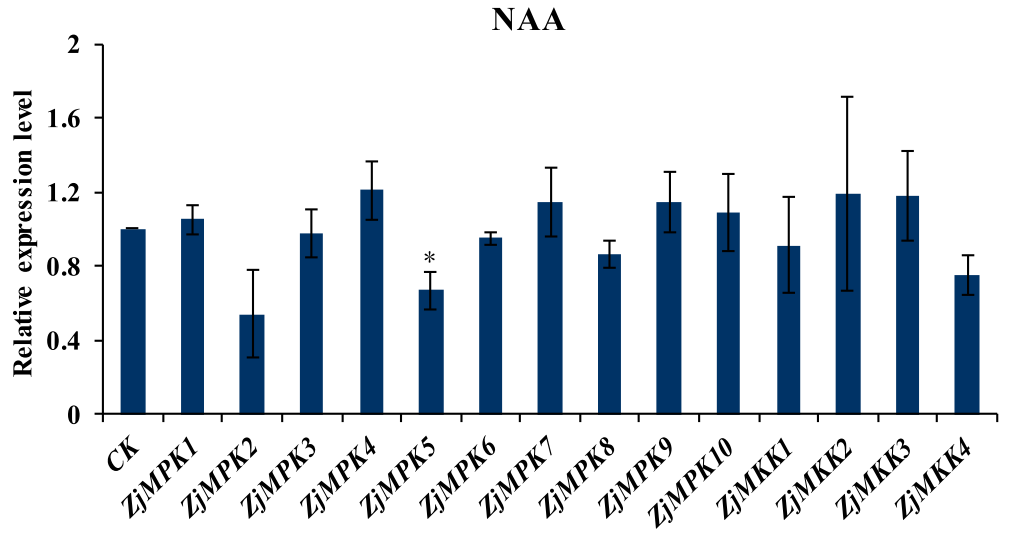

$Z j M P K$ and $Z j M K K$ genes

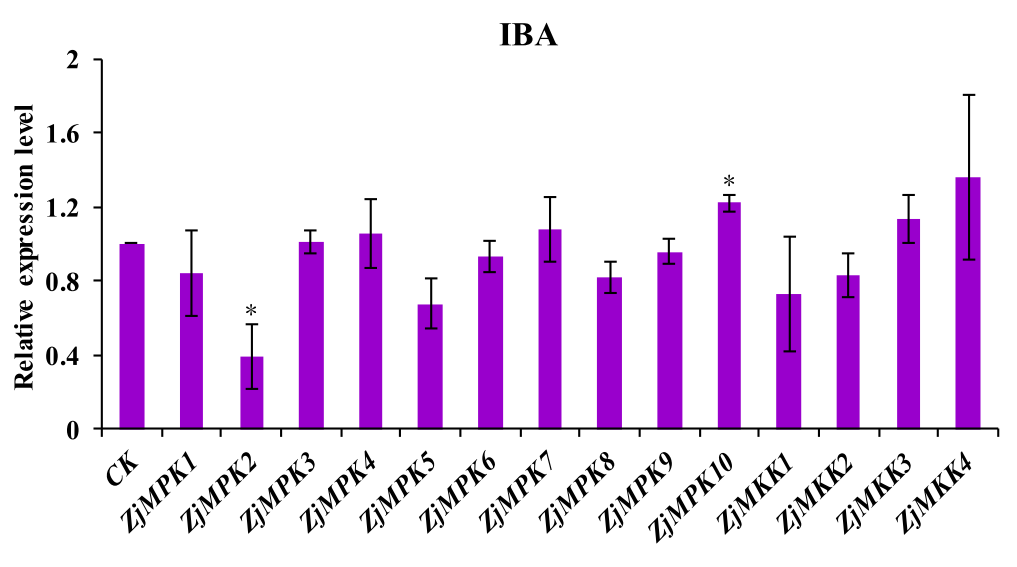

$Z j M P K$ and $Z j M K K$ genes

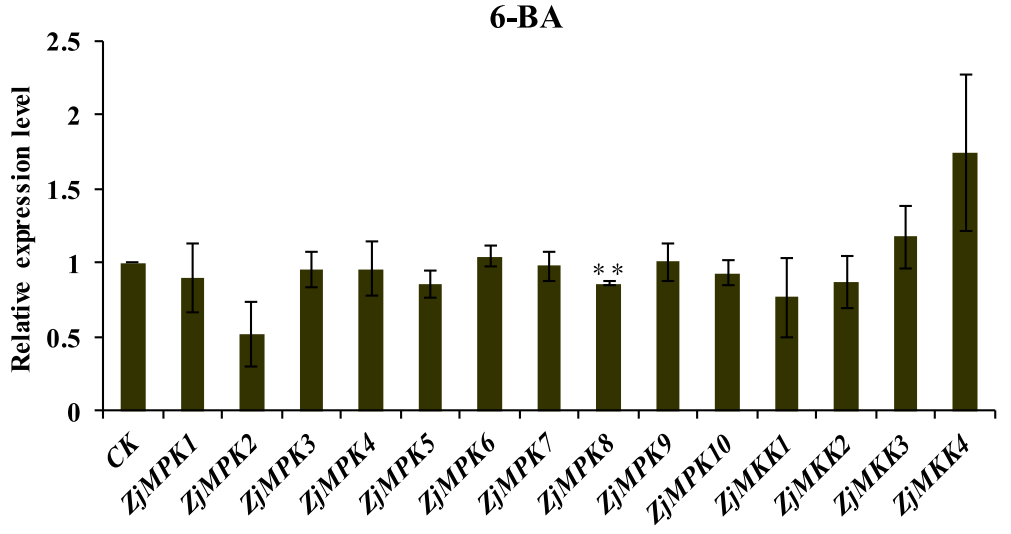

$Z j M P K$ and $Z j M K K$ genes

Fig. 9 Relative expression of ZjMPK and ZjMKK genes analyzed by qRT-PCR upon plant growth regulators treatment. ZjACT primers were used as the internal standard for each gene. The mean expression value was calculated from 3 independent replicates. The vertical bars indicate the standard deviation. Significant and highly significant difference are shown as * $(P<0.05)$ and ${ }^{* *}(P<0.01)$, respectively

MAPKKs in apple, and so on $[8,10-13,31]$. However, no data set of MAPKs and MAPKKs is available for Chinese jujube. In this study, a comprehensive analysis of gene structure, phylogenetic relationship, chromosomal distribution and expression of ZjMAPKs and
ZjMAPKKs were presented at genome level. A total of 10 MAPKs and 5 MAPKKs were first identified, and their proteins property, such as the isoelectric point and molecular weight, were also predicted. When comparing the structure of ZjMAPKs genes with other plants, the 


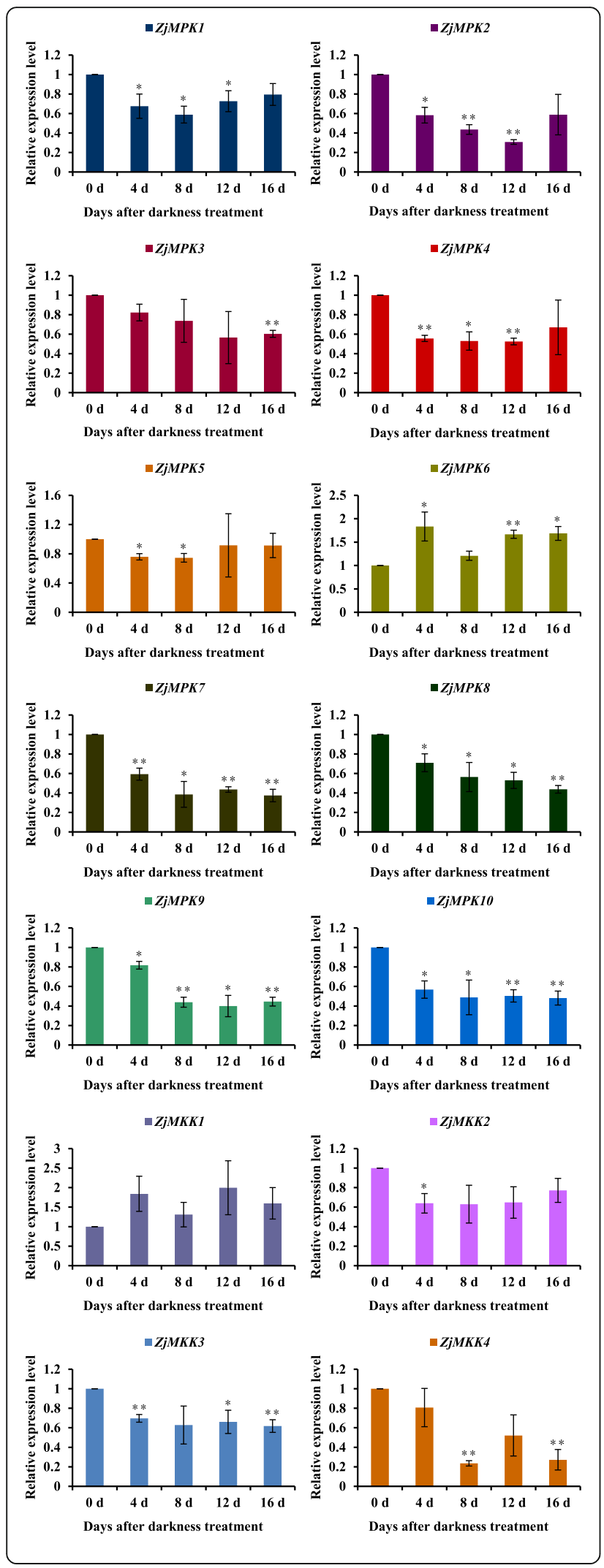

Fig. 10 Relative expression of ZjMPK and ZjMKK genes analyzed by qRT-PCR upon darkness treatment. ZjACT primers were used as the internal standard for each gene. The mean expression value was calculated from 3 independent replicates. The vertical bars indicate the standard deviation. Significant and highly significant difference are shown as * $(P<0.05)$ and ${ }^{*}(P<0.01)$, respectively

entire group A ZjMAPKs consisted of six exons in jujube, Arabidopsis and mulberry. Group B genes contained six exons in jujube, mulberry, tomato and poplar, while AtMPK5, AtMPK11 and AtMPK13 consisted of four exons in Arabidopsis. Group C ZjMAPKs consisted of only two exons with highly conserved sizes, which is similar to the other plants, including Arabidopsis, poplar and apple. Group D ZjMAPKs were complex in jujube, Arabidopsis, tobacco, poplar and apple, despite having the same number of exons. Chinese jujube and mulberry have the same total number genes which less than the other dicotyledons (Table 2), the main reason might be due to they do not experience the related whole genome duplication in the evolutionary process.

To date, more and more evidences suggested that the MAPK cascades are involved in biotic and abiotic stress response. The best characterized MAPKs are AtMPK3, AtMPK4 and AtMPK6 in Arabidopsis and their orthologs in other plant species, all of which are mainly activated by different stimulus including abiotic stresses, pathogens and oxidative stress [32]. It has been indicated that MAPK proteins classified in the same groups might serve similar functions in different species [10]. Based on sequence homology, MAPKs can be classified into four distinct subfamilies (group A, B, C and D) in plant. In Arabidopsis thaliana, AtMPKs of group A have been reported to be positive regulators of defense signaling [33], especially AtMPK3 and AtMPK6 have been described to mediate innate immunity [34]. In our present study, we found that ZjMPK1 which belong to Group A was expressed at a markedly lower level in the diseased plantlets infected by phytoplasma (Fig. 11), and the phylogenetic tree (Fig. 5) indicates that AtMPK3, 6 and ZjMPK1 have close phylogenetic relationship. So we can infer the potential functions of ZjMPK1 from the known AtMPKs in Arabidopsis, that ZjMPK1 might play an important role in the process of plant-phytoplasma interaction.

Most plant MAPK cascade genes were response to abiotic and biotic stress, and a few genes have been proved to be involved in reproductive organ development. Arabidopsis AtMPK6 was involved in anther and embryo development [35]. Guan et al. also proved that AtMPK3/ AtMPK6 in Arabidopsis were necessary to guide the direction of pollen tubes, but not in their growth [36]. Moreover, A MAPKKK gene has been successfully isolated from Solanum chacoense which involved in embryo 


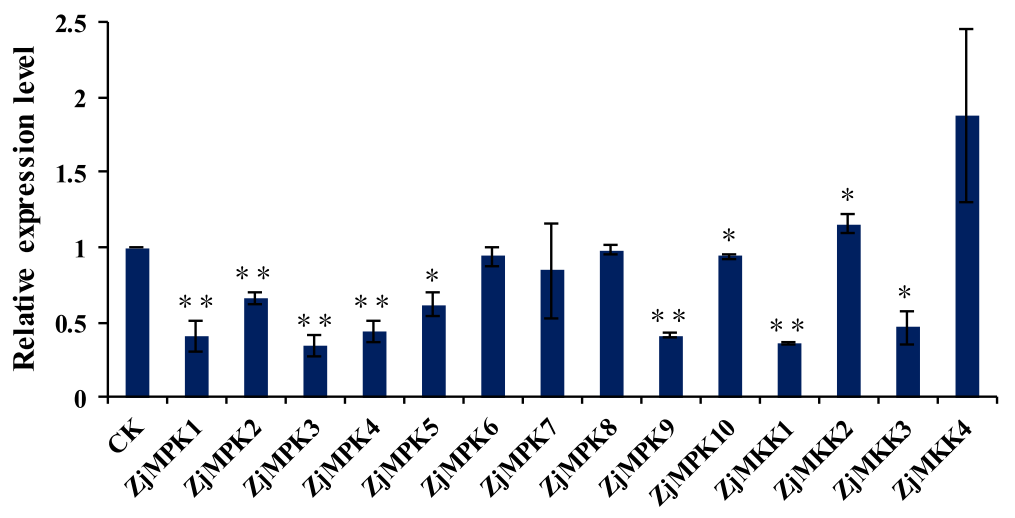

ZjMPK and ZjMKK genes

Fig. 11 Relative expression of ZjMPK and ZjMKK genes analyzed by qRT-PCR under phytoplasma infection. ZjACT primers were used as the internal standard for each gene. The mean expression value was calculated from 3 independent replicates. The vertical bars indicate the standard deviation. Significant and highly significant difference are shown as * $(P<0.05)$ and $* *(P<0.01)$, respectively

sac and pollen development [37]. Another research of genome-wide analysis showed that one expansion class of MAPKKKs in solanaceous species played some specific roles in reproductive organ development [38]. Interestingly, in this study, ZjMKK5 (homolog with MKK9 in Arabidopsis and other plants) was specific expressed in early stage of flower bud development, indicating its function is related to jujube flower development. However, previously there was no evidence that MKK9 homolog was involved in reproductive organ development. Among MAPK cascade, both MAPKKKs and MAPKs were reported to play roles in reproductive organ development, some MAPKKs must mediate in their process. We deduced that MKK9 just mediated this process. MKK9 directly phosphorylates and activates MPK6 [39-41], which was proved to involve in anther, pollen and embryo development $[35,36]$. Hence how the ZjMKK5 cascade regulates reproductive organ development in Chinese jujube need to elucidate by further studies.

\section{Conclusions}

Ten MAPK and five MAPKK genes were identified from the genome database of Chinese jujube, and then a series of bioinformatics analysis were conducted. The expression analysis of ZjMAPK and ZjMAPKK genes using RT-PCR shown that majority of ZjMAPK and ZjMAPKK genes were expressed in all tested organs/tissues with considerable differences. Moreover, ZjMKK5 was specific expressed in early development stage of jujube flower bud, indicating it plays some roles in reproductive organs development. The transcript expression results demonstrated that most ZjMAPK and ZjMAPKK genes were down-regulated in response to plant growth regulators, darkness treatment and phytoplasma infection.

Taken together, in this work we presented a comprehensive analysis of the ZjMPK and ZjMKK gene family.
It would provide a better understanding of the complexity of MAPKs in plants and provide an important foundation for further functional study of MAPK and MAPKK family genes in Chinese jujube.

\section{Additional files}

Additional file 1: Figure S1. Different stages of floral organ development I: Small bud 1, diameter 0.5 1.0 mm; II: Small bud 2, diameter 1.0 1.5 mm; II: Middle bud, diameter 1.5 2.0 mm; IV: Big bud, diameter 2.0 2.5 mm; V: Yellow bud, diameter 2.5 3.0 mm; VI: Split bud, diameter $3.0 \sim 3.5 \mathrm{~mm}$; VII: Full flower, diameter 3.5 5 mm. (PDF $125 \mathrm{~kb}$ )

Additional file 2: All sequence data used in this manuscript. (ZIP $36 \mathrm{~kb}$ ) Additional file 3: Table S1. The primer information of ZjMAPKs and ZjMAPKKs. (DOCX $17 \mathrm{~kb}$ )

Additional file 4: Figure S2. Relative expression of ZjMKK5 gene analyzed by qRT-PCR in Chinese jujube floral organ at different development stages. ZjACT primers were used as the internal standard. The mean expression value was calculated from 3 independent replicates. The vertical bars indicate the standard deviation. Different lowercase and uppercase letters were defined as significant and highly significant difference, respectively. (PDF 93 kb)

\section{Abbreviations}

6-BA: 6-benzyladenine; CDS: Coding sequence; GSDS: Gene Structure Display Server; IBA: Indole-3-butyric acid; JWB: Jujube witches' broom; MAPK: Mitogen-activated protein kinases; MAPKK: Mitogen-activated protein kinases kinases; MS: Murashige and Skoog; NAA: a-Naphthalene acetic acid; NCBI: National Center for Biotechnology Information; NJ: Neighbor-joining; ORF: Open reading frame; qRT-PCR: Quantitative real-time PCR; RT-

PCR: Reverse transcription PCR

\section{Acknowledgements \\ Not applicable.}

\section{Funding}

Funds for Hebei Distinguished Young Scholar (C2016204145); Youth Fund of Hebei Province Natural Science Foundation (C2016204157); National Science and Technology Support Plan of China (2013BAD14B03); Agricultural University of Hebei Foundation for Leaders of Disciplines in Science Technology.

\section{Availability of data and materials}

All data and materials are presented in the main paper and additional file. Seven different development stages of floral organs can be found in Additional file 1: 
Figure S1. All sequence data used in this manuscript was summarized in Additional file 2. The primers used in this study are available in Additional file 3 : Table S1. ZjMKK5 gene expression in Chinese jujube floral organ was shown in Additional file 4: Figure S2.

\section{Authors' contributions}

$J Z$ and $M L$ designed the research and wrote the paper. $Z L$ and $L Z$ performed the experiments, analyzed the data and wrote the paper. CX and HF participated in the data analysis. All authors read and approved the final the manuscript.

\section{Ethics approval and consent to participate}

The healthy and diseased jujube trees used in this study were from the Experimental Station of Chinese Jujube, Hebei Agricultural University, in Baoding, Hebei. Chinese jujube is one of traditional and widespread fruit trees in China, and it is not an endangered species. No specific permits are required for sample collection on Chinese jujube.

\section{Consent for publication}

Not applicable.

\section{Competing interests}

The authors declare that they have no competing interests.

\section{Publisher's Note}

Springer Nature remains neutral with regard to jurisdictional claims in published maps and institutional affiliations.

\section{Author details}

${ }^{1}$ Research Center of Chinese Jujube, Hebei Agricultural University, Baoding, China. ${ }^{2}$ College of Life Science, Hebei Agricultural University, Baoding, China. ${ }^{3} \mathrm{BGI}-S h e n z h e n$, Shenzhen, China.

Received: 16 March 2017 Accepted: 1 November 2017

Published online: 09 November 2017

\section{References}

1. Tena G, Asai T, Chiu WL, et al. Plant mitogen-activated protein kinase signaling cascades. Curr Opin Plant Biol. 2001;4(5):392-400.

2. Pedley KF, Martin GB. Role of mitogen-activated protein kinases in plant immunity. Curr Opin Plant Biol. 2005;8(5):541-7.

3. Qiu JL, Zhou L, Yun BW, et al. Arabidopsis mitogen-activated protein kinase kinases MKK1 and MKK2 have overlapping functions in defense signaling mediated by MEKK1, MPK4, and MKS1. Plant Physiol. 2008;148(1):212-22.

4. Wang J, Pan C, Wang Y, et al. Genome-wide identification of MAPK, MAPKK, and MAPKKK gene families and transcriptional profiling analysis during development and stress response in cucumber. BMC Genomics. 2015;16(1):386.

5. Xu J, Zhang S. Mitogen-activated protein kinase cascades in signaling plant growth and development. Trends Plant Science. 2015;20(1):56-64.

6. Zhang $T$, Liu Y, Yang $T$, et al. Diverse signals converge at MAPK cascades in plant. Plant Physiol Biochem. 2006;44(5):274-83.

7. Ichimura K, Shinozaki K, Tena G, et al. Mitogen-activated protein kinase cascades in plants: a new nomenclature. Trends Plant Sci. 2002;7(7):301-8.

8. Colcombet J, Hirt H. Arabidopsis MAPKs: A complex signalling network involved in multiple biological processes. Biochem J. 2008;413(2):217-26.

9. Liu Y, Zhang D, Wang $L$, et al. Genome-wide analysis of mitogen-activated protein kinase gene family in maize. Plant Mol Biol Report. 2013;31(6):1446-60.

10. Nicole MC, Hamel LP, Morency MJ, et al. MAP-ping genomic organization and organ-specific expression profiles of poplar MAP kinases and MAP kinase kinases. BMC Genomics. 2006;7(1):223.

11. Rao KP, Richa T, Kumar K, et al. Silico analysis reveals 75 members of mitogen-activated protein kinase kinase kinase gene family in rice. DNA Res. 2010;17(3):139-53.

12. Chen L, Hu W, Tan S, et al. Genome-wide identification and analysis of MAPK and MAPKK gene families in Brachypodium distachyon. PLoS One. 2012;7(10):e46744.

13. Zhang S, Xu R, Luo X, et al. Genome-wide identification and expression analysis of MAPK and MAPKK gene family in Malus domestica. Gene. 2013;531:377-87.

14. Zhang $X$, Cheng T, Wang G, et al. Cloning and evolutionary analysis of tobacco MAPK gene family. Mol Biol Rep. 2013;40(2):1407-15.
15. Wang G, Lovato A, Polverari A, et al. Genome-wide identification and analysis of mitogen activated protein kinase kinase kinase gene family in grapevine (Vitis vinifera). BMC Plant Biol. 2014;14:219.

16. Zhang $X$, Wang $L$, Xu X, et al. Genome-wide identification of mitogenactivated protein kinase gene family in Gossypium raimondii and the function of their corresponding orthologs in tetraploid cultivated cotton. BMC Plant Biol. 2014;14:345.

17. Wei C, Liu X, Long D, et al. Molecular cloning and expression analysis of mulberry MAPK gene family. Plant Physiol Biochem. 2014;77:108-16.

18. Lu K, Guo W, Lu J, et al. Genome-wide survey and expression profile analysis of the mitogen-activated protein kinase (MAPK) gene family in Brassica rapa. PLoS One. 2015;10(7):e0132051

19. Hamel LP, Nicole MC, Sritubtim S, et al. Ancient signals: comparative genomics of plant MAPK and MAPKK gene families. Trends Plant Sci. 2006;1 1(4):192-8.

20. Beckers GJM, Jaskiewicz M, Liu Y, et al. Mitogen-activated protein kinases 3 and 6 are required for full priming of stress responses in Arabidopsis thaliana. Plant Cell. 2009;21(3):944-53.

21. Jammes F, Song C, Shin D, et al. MAP kinases MPK9 and MPK12 are preferentially expressed in guard cells and positively regulate ROS-mediated ABA signaling. Proc Natl Acad Sci. 2009;106(48):20520-5.

22. Shi J, Zhang L, An H, et al. GhMPK16, a novel stress-responsive group D MAPK gene from cotton, is involved in disease resistance and drought sensitivity. BMC Mol Biol. 2011;12(1):22.

23. Qu ZZ, Wang YH. China fruit's monograph-Chinese jujube volume. Beijing: China Forestry Publishing House; 1993.

24. Liu M. Chinese jujube: botany and horticulture. Hortic Rev. 2010:32:229.

25. Liu MJ, Zhao J, Cai QL, et al. The complex jujube genome provides insights into fruit tree biology. Nat Commun. 2014; doi:10.1038/ncomms6315.

26. Letunic I, Copley RR, Schmidt S, et al. SMART 4.0: towards genomic data integration. Nucleic Acids Res. 2004;32:D142-4.

27. Yan DH, Xia X, Yin W. NF-YB family genes identified in a poplar genomewide analysis and expressed in Populus euphratica are responsive to drought stress. Plant Mol Biol Report. 2013:31(2):363-70.

28. Zhang $L$, Jin Z, Feng $C$, et al. Genome-wide identification, characterization of the MADS-box gene family in Chinese jujube and their involvement in flower development. Sci Rep. 2017;7(1):1025

29. Kumar S, Stecher G, Peterson D, et al. MEGA-CC: computing core of molecular evolutionary genetics analysis program for automated and iterative data analysis. Bioinformatics. 2012;28(20):2685-6.

30. Livak KJ, Schmittgen TD. Analysis of relative gene expression data using real-time quantitative PCR and the 2(-Delta Delta C(T)) method. Methods 2001:25(4):402-8.

31. Kong F, Wang J, Cheng L, et al. Genome-wide analysis of the mitogenactivated protein kinase gene family in Solanum lycopersicum. Gene. 2012;499(1):108-20.

32. Pitzschke A, Schikora A, Hirt H. MAPK Cascade signalling networks in plant defence. Curr Opin Plant Biol. 2009;12(4):421-6.

33. Romeis T, Ludwig AA, Martin $R$, et al. Calcium-dependent protein kinases play an essential role in a plant defence response. EMBO J. 2001;20(20):5556-67.

34. Nakagami H, Pitzschke A, Hirt H. Emerging MAP kinase pathways in plant stress signalling. Trends Plant Science. 2005;10:339-46.

35. Bush SM, Krysan PJ. Mutational evidence that the Arabidopsis MAP kinase MPK6 is involved in anther, inflorescence, and embryo development. J Exp Bot. 2007:58(8):2181-91.

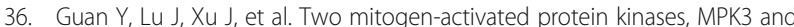
MPK6, are required for funicular guidance of pollen tubes in Arabidopsis. Plant Physiol. 2014;165(2):528-33.

37. Lafleur E, Kapfer C, Joly V, et al. The FRK1 mitogen-activated protein kinase kinase kinase (MAPKKK) from Solanum chacoense is involved in embryo sac and pollen development. J Exp Bot. 2015;66(7):1833-43.

38. Daigle C, Matton DP. Genome-wide analysis of MAPKKKs shows expansion and evolution of a new MEKK class involved in solanaceous species sexual reproduction. BMC Genomics. 2015;16(1):1037.

39. Zhou C, Cai Z, Guo Y, et al. An arabidopsis mitogen-activated protein kinase cascade, MKK9-MPK6, plays a role in leaf senescence. Plant Physiol. 2009;150:167-77.

40. Lampard GR, Wengier DL, Bergmann DC. Manipulation of mitogen-activated protein kinase kinase signaling in the Arabidopsis stomatal lineage reveals motifs that contribute to protein localization and signaling specificity. Plant Cell. 2014;26(8):3358-71.

41. Lei L, Li Y, Wang Q, et al. Activation of MKK9-MPK3/MPK6 enhances phosphate acquisition in Arabidopsis thaliana. New Phytol. 2014;203(4):1146-60. 